\title{
The Influence of the Reaction Conditions \\ on the Size of Silver Nanoparticles \\ in Carey Lea's Concentrated Sols
}

\author{
Sergey A. Vorobyeva*, \\ Maksim N. Likhatski ${ }^{a}$, Alexander S. Romanchenko ${ }^{a}$, \\ Timur Y. Ivanenko ${ }^{b}$, Diana A. Masharovab, \\ Mikhail N. Volochaev ${ }^{c, d}$ and Yuri L. Mikhlin ${ }^{\mathrm{a}}$ \\ anstitute of Chemistry and Chemical Technology SB RAS \\ FRC "Krasnoyarsk Science Center SB RAS" \\ Krasnoyarsk, Russian Federation \\ ${ }^{b}$ Siberian Federal University \\ Krasnoyarsk, Russian Federation \\ ${ }^{c}$ Kirensky Institute of Physics SB RAS \\ Krasnoyarsk, Russian Federation \\ ${ }^{d}$ Reshetnev Siberian State Aerospace University \\ Krasnoyarsk, Russian Federation
}

Received 11.06.2020, received in revised form 22.06.2020, accepted 23.08.2020

Abstract. The reaction of reduction solution of $\mathrm{Ag}$ (I) by Fe (II) citrate complex was studied herein. This allows you to receive silver nanoparticles with high stability with a concentration above $60 \mathrm{~g} / 1$. It was determined that the nanoparticles size depends on the injection rate, mixing rate, reagent concentration, $\mathrm{pH}$ and some post-synthetic operations on the average size of nanoparticles. It was shown that decreasing the concentration of $\mathrm{Ag}$ (I) and increasing the concentration of stabilizer also bringing $\mathrm{pH}$ to 7 lead to small and uniform particles. Optimal conditions were found that made it possible to reduce particle size and reduce the concentration of reagents by $33 \%$ in the results. According to XPS, TEM, DLS and FTIR datas, nanoparticles of metallic silver with a size of $6.5 \pm 1.8 \mathrm{~nm}$ were obtained, which stabilized by the product of partial decay of the citrate ion.

Keywords: silver nanoparticles, concentrated sols, influence of reaction conditions, citrate ion.

(C) Siberian Federal University. All rights reserved

This work is licensed under a Creative Commons Attribution-NonCommercial 4.0 International License (CC BY-NC 4.0).

* Corresponding author E-mail address: yekspatz@ya.ru 
Citation: Vorobyev S.A., Likhatski M.N., Romanchenko A.S., Ivanenko T.Y., Masharova D.A., Volochaev M.N., Mikhlin Yu.L. The influence of the reaction conditions on the size of silver nanoparticles in carey lea's concentrated sols, J. Sib. Fed. Univ. Chem., 2020, 13(3), 372-384. DOI: 10.17516/1998-2836-0190

\title{
Влияние реакционных условий \\ на размер наночастиц серебра \\ в концентрированных золях Carey Lea
}

\author{
С.А. Воробьев ${ }^{\mathrm{a}}$, М.Н. Лихацкий \\ А.С. Романченко ${ }^{a}$, Т.Ю. Иваненко ${ }^{\sigma}$,

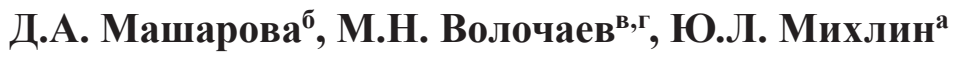 \\ ${ }^{a}$ Институт химии и химической технологии \\ ФИЦ «Красноярский научный центр СО РАН» \\ Российская Федерация, Красноярск \\ ${ }^{6}$ Сибирский федеральный университет \\ Российская Федераиия, Красноярск \\ ${ }^{6}$ Институт физики им. Киренского, КНЦ СО РАН \\ Российская Федераиия, Красноярск \\ ${ }^{2}$ Сибирский государственный университет науки \\ и технологий им. М.Ф. Решетнева \\ Российская Федерация, Красноярск
}

Аннотация. В данной работе был изучен процесс восстановления растворов $\mathrm{Ag}$ (I) цитратными комплексами $\mathrm{Fe}$ (II), который позволяет получать наночастицы серебра с высокой стабильностью и концентрацией более 60 г/л. В ходе работы было установлено влияние скорости введения, скорости перемешивания, концентрации реагентов, $\mathrm{pH}$ среды и некоторых постсинтетических операций на средний размер наночастиц. Показано, что снижение концентрации $\mathrm{Ag}(\mathrm{I})$ и повышение концентрации стабилизатора, доведение $\mathrm{pH}$ реакционной среды до 7 позволяют получать наиболее мелкие и однородные частицы. В результате были найдены оптимальные условия, которые дали возможность уменьшить размер частиц и вместе с тем снизить концентрацию реактивов на 33 \%. По данным РФЭС, ПЭМ, DLS и ИК были получены наночастицы металлического серебра с размером $6.5 \pm 1.8$ нм, стабилизированные продуктом частичного распада цитрат-иона.

Ключевые слова: наночастицы серебра, концентрированные золи, влияние реакционных условий, цитрат-ион.

Цитирование: Воробьев, С.А. Влияние реакционных условий на размер наночастиц серебра в концентрированных золях Carey Lea / С.А. Воробьев, М.Н. Лихацкий, А.С. Романченко, Т.Ю. Иваненко, Д.А. Машарова, М.Н. Волочаев, Ю.Л. Михлин // Журн. Сиб. федер. ун-та. Химия, 2020. 13(3). С. 372-384. DOI: 10.17516/1998-2836-0190 


\section{Введение}

Наночастицы (НЧ) серебра могут быть использованы для производства гетерогенных катализаторов, антимикробных материалов, оптических систем, электронных и сенсорных устройств, фотонной техники, композитных материалов и структур, 2D- и 3D-печати, SERS и т.д. [1, 2]. Существует масса способов получения НЧ серебра, однако наиболее распространен т.н. мокрый химический синтез, где растворы солей серебра в присутствии стабилизаторов обрабатывают растворами химических восстановителей [3]. Данный метод относительно прост и позволяет контролировать размер, форму и состав частиц [3]. Однако ввиду склонности частиц к агрегации и укрупнению синтез необходимо проводить в разбавленных растворах. В результате чего выход наночастиц, как правило, составляет 0.05-1.0 г/л, что требует дополнительных процедур для концентрирования, выделения и очистки наночастиц, а также утилизации отработанных растворов $[4,5]$. Протокол синтеза может требовать использования дорогих и токсичных реагентов [6-8]. Кроме того, для стабилизации наночастиц часто в больших концентрациях используют поверхностно-активные вещества с высокой молекулярной массой, которые необратимо блокируют поверхность частиц, что может быть неприемлемым для многих областей их применения $[9,10]$.

Исключением является простой метод синтеза гидрозолей серебра с концентрацией до 60 г/л, предложенный ученым Carey Lea (Кэри Ли) 130 лет назад, где нитрат серебра восстанавливают раствором цитратного комплекса железа (II) [11]. Образующиеся в гидрозоле наночастицы серебра размером 6-15 нм демонстрируют высокую коллоидную стабильность и могут быть многократно диспергированы после коагуляции электролитом. В типичной процедуре Carey Lea молярное отношение $\mathrm{AgNO}_{3}: \mathrm{FeSO}_{4}: \mathrm{Na}_{3}$ Cit составляет $1.0: 1.8: 3.2$. Таким образом, методика синтеза Кэри Ли могла бы быть использована для простого и недорогого промышленного производства наночастиц серебра с заданными характеристиками. Однако, несмотря на продолжительную историю и уникальность, эта система осталась практически неизученной. Отсутствуют исчерпывающие данные о влиянии условий реакции на размер наночастиц и их стабильность. Кроме того, методика синтеза Кэри Ли неизменна с момента ее разработки, практически не изучалось влияние условий реакции и постреакционных манипуляций на размер частиц.

На данный момент существует ряд исследований по кинетике агрегации [12-14], структуре, химической активности защитной оболочки $[12,14,15]$ и практическому применению наночастиц Кэри Ли для производства электро- и теплопроводящих паст [16-19]. Однако нам известна только одна работа [20], где изучали влияние некоторых условий реакции на размер и стабильность частиц. Авторы синтезировали гомогенные наночастицы с размером 20 нм при скорости перемешивания 1410 об/мин и молярном отношении $\mathrm{AgNO}_{3}: \mathrm{FeSO}_{4}: \mathrm{Na}_{3} \mathrm{Cit}=1.0: 1.8: 4.8$, что даже больше, чем в классической рецептуре Кэри Ли. Кроме того, как показал литературный обзор и наши предварительные эксперименты, синтез наночастиц методом Кэри Ли плохо воспроизводим. Часто авторы добавляют реагенты вручную с разными скоростями, в различные части реакционной ячейки и под разными углами, используемые магнитные мешалки не калиброваны, а температура синтеза равна температуре окружающей среды и не контролируется.

Задача данной работы состояла в создании установки для синтеза наночастиц методом Кэри Ли в контролируемых и воспроизводимых условиях, в изучении влияния различных фак- 
торов на размер и стабильность наночастиц серебра, нахождении оптимальных условий синтеза частиц и их исследовании физико-химическими методами.

\section{Экспериментальная часть}

Все использованные реактивы были аналитической чистоты и дополнительной очистке не подвергались. Растворы реагентов готовили непосредственно перед проведением экспериментов с применением деионизированной воды (электропроводность менее 15 мкСм).

Синтез наночастиц серебра проводили по модифицированной методике Кэри Ли [11] при контролируемой температуре, скорости впрыска и скорости перемешивания в специально собранном и откалиброванном устройстве (рис. 1). Устройство содержало блок ввода раствора $\mathrm{AgNO}_{3}$, приводимый в действие Nema 23HS5628, и реакционную ячейку с перемешиванием шаговым двигателем Nema 17HS2408. Ошибка объема впрыска $\mathrm{AgNO}_{3}$ составляла 0.5-0.9\%, скорости впрыска $\mathrm{AgNO}_{3}$ 0.6-0.8\% и скорости перемешивания 0.1-0.3\%. Оба блока реакционной установки были термостатированы с использованием криотермостата FT-211-25 $\left(25.0 \pm 0.2{ }^{\circ} \mathrm{C}\right)$.

В реакционную ячейку загружали водные растворы $\mathrm{Na}_{3} \mathrm{Cit}\left(0.6-1.8 \mathrm{M} ; 5\right.$ мл), $\mathrm{FeSO}_{4}$ (0.6$0.9 \mathrm{M} ; 5$ мл) и $\mathrm{NH}_{3} \cdot \mathrm{H}_{2} \mathrm{O}(12 \mathrm{M} ; 0.0-0.25$ мл) и термостатировали при температуре эксперимента $25{ }^{\circ} \mathrm{C}$ и скорости перемешивания $100-1000$ об/мин в течение 5 мин, далее производили инжекцию раствора $\mathrm{AgNO}_{3}$ (0.05 - 0.6 M; 5 мл) со скоростью 1.0-25.0 мл/с, после чего реакционную смесь продолжали перемешивать еще в течение 5 мин. После смешивания реагентов наблюдалось осаждение черно-коричневого осадка агрегированных наночастиц серебра:

$$
\mathrm{FeSO}_{4}+\mathrm{Na}_{3} \mathrm{Cit}+\mathrm{AgNO}_{3} \rightarrow \mathrm{FeCit}+\mathrm{Na}_{2} \mathrm{SO}_{4}+\mathrm{NaNO}_{3}+\mathrm{Ag} \downarrow
$$

Для очистки полученный осадок собирали и центрифугировали в течение 10 мин с ускорением $1-600 \mathrm{~g}$, осадок отделяли и растворяли в 5 мл воды, после чего добавляли раствор $\mathrm{NaNO}_{3}(0.2-1.0 \mathrm{M} ; 5$ мл) для осаждения частиц, снова центрифугировали и осадок растворя-

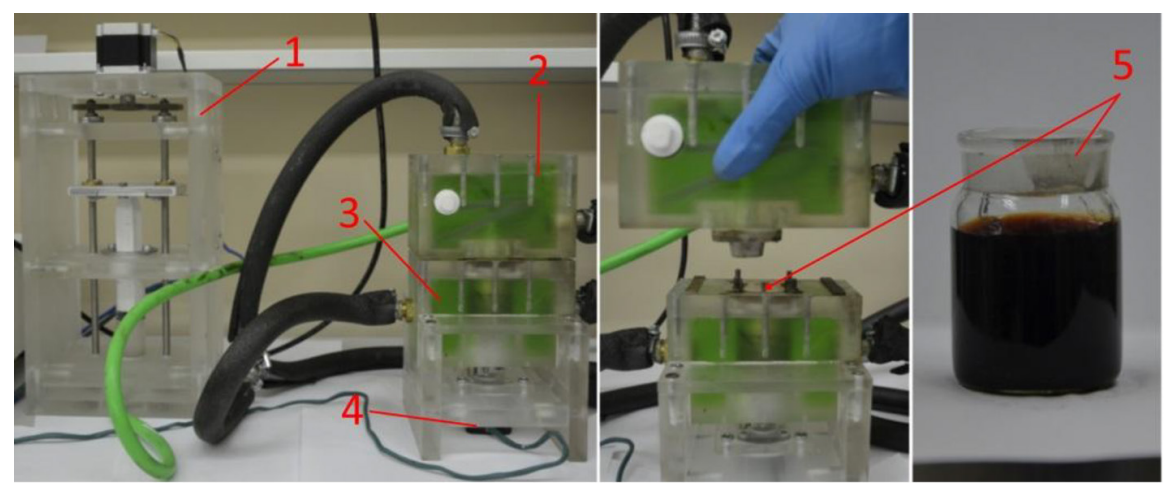

Рис. 1. Внешний вид установки для синтеза наночастиц серебра при контролируемых условиях, содержащий: дозирующий блок -1 , термостатированные инжекционный -2 и реакционный блоки -3 , магнитную мешалку -4 и реакционную ячейку -5

Fig. 1. Facility for the synthesis of silver nanoparticles under controlled conditions and a reaction cell with prepared silver sol consisted of dosing unit -1 ; thermostatically injection and reaction blocks $-2,3$; magnetic stirrer -4 ; reaction cell -5 
ли в воде. Полученный таким образом очищенный гидрозоль серебра был подвергнут дальнейшему изучению.

Рентгеновские фотоэлектронные спектры (РФЭС) были записаны на спектрометре SPECS (SPECS, Германия), оборудованном анализатором PHOIBOS150 MCD-9 с использованием для возбуждения монохроматического излучения $\mathrm{Al} \mathrm{K}_{\alpha}(1486.6$ эB). Давление в аналитической камере было $10^{-9}$ мБар. Исследования методом динамического рассеяния света (DLS) проводили с помощью спектрометра Zetasizer Nano ZS (Malvern, Великобритания) под углом рассеяния $173^{\circ}$ в поликарбонатной ячейке при температуре $25^{\circ} \mathrm{C}$. По данным DLS были определены средние гидродинамические размеры частиц (далее средний размер частиц), которые использовали для нахождения влияния реакционных условий на исследуемую систему. Микрофотографии наночастиц были получены на электронном просвечивающем микроскопе HT7700 (Hitachi, Япония) с ускоряющим напряжением 110 кВ. ИК-Фурье спектры были записаны для порошков наночастиц серебра в таблетках KBr с помощью Фурье-спектрометра Vector 22 (Bruker, Германия).

\section{Результаты и их обсуждение}

Как правило, полученные наночастицы Кэри Ли [11-19] для очистки осаждают 1.0 M раствором $\mathrm{NaNO}_{3}$, центрифугируют, надосадочный раствор сливают, а осадок пептизируют в деионизованной воде, процедуру повторяют до 3 раз. Нами было изучено влияние концентрации коагулянта $\mathrm{NaNO}_{3}(0.2-1.0 \mathrm{M})$ и ускорения центрифугирования $(1-600 \mathrm{~g})$ на размер выделенных наночастиц.

На рис. $2 a$ видно, что при увеличении концентрации коагулянта средний размер частиц постепенно снижается. Видимо, при низкой концентрации $\mathrm{NaNO}_{3}$ происходит сначала осаждение наиболее крупных и менее стабильных частиц, однако с ростом концентрации коагулянта осаждаются все более мелкие и стабильные частицы.

На рис. 26 представлена зависимость размера частиц от ускорения центрифугирования. При ускорении $1 \mathrm{~g}$ происходит неполное отделение электролита, что, вероятнее всего, приво-
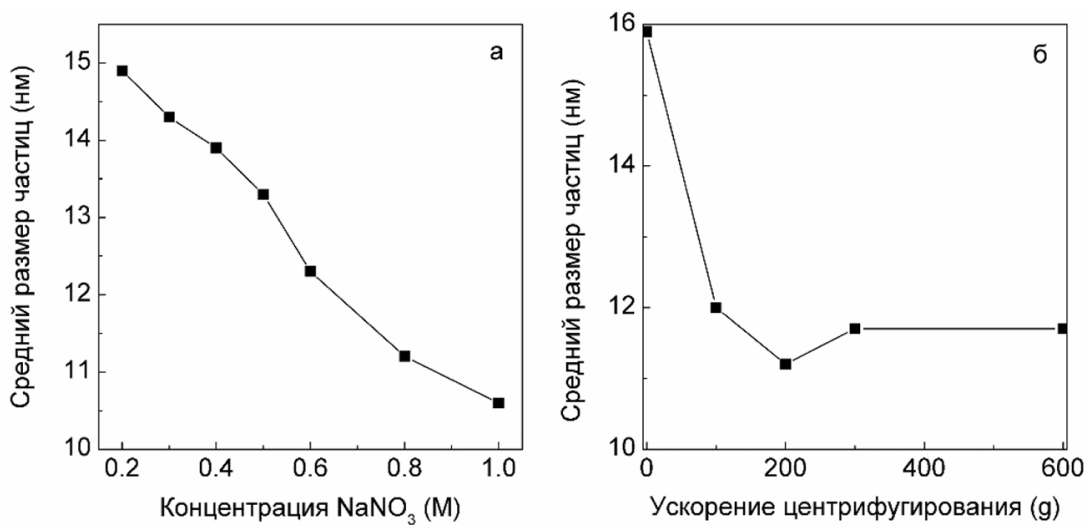

Рис. 2. Влияние концентрации $\mathrm{NaNO}_{3}$ (а) и (б) ускорения центрифугирования на средний размер частиц после очистки

Fig. 2. Effect of the concentration of $\mathrm{NaNO}_{3}$ (a) and on centrifugation acceleration (б) on average particle size after nanoparticle purification 
дит к электролитической агломерации частиц. Таким образом, рост ускорения центрифугирования до $200 \mathrm{~g}$ ведет к ожидаемому снижению размера частиц, однако при больших значениях ускорения наблюдаем увеличение размера частиц, связанное со сращиванием частиц при центрифугировании. Установленные оптимальные условия очистки (концентрация $\mathrm{NaNO}_{3}=1.0 \mathrm{M}$; ускорение центрифугирования $=200 \mathrm{~g}$ ), минимально влияющие на систему, были использованы для всех дальнейших опытов.

На рис. 3 приведены зависимости влияния скорости инжекции раствора $\mathrm{AgNO}_{3}(1.0$ 25.0 мл/с) и скорости перемешивания реакционной среды (100 - 1000 об/мин) на средний размер частиц. Видно (рис. $3 a$ ), что увеличение скорости инжекции раствора $\mathrm{AgNO}_{3}$ с 1 до 5 мл/с вызывает резкое снижение среднего размера частиц, после чего зависимость выходит на плато. Увеличение скорости перемешивания реакционной среды (рис. 3б) приводит к закономерному снижению среднего размера частиц. Высокие скорости перемешивания растворов и инжекции реагентов позволяют создать более однородные условия по всему реакционному объему и, как следствие, получить более мелкие и однородные частицы. Таким образом, далее для синтеза наночастиц серебра мы использовали максимальные скорости инжекции $\mathrm{AgNO}_{3}$ (25.0 мл/с) и скорости перемешивания реакционной среды (1000 об/мин).

Концентрации стабилизатора и восстановителя имеют ключевые значения для синтеза наночастиц металлов. В классической рецептуре Кэри Ли молярное отношение $\mathrm{AgNO}_{3}: \mathrm{FeSO}_{4}$ : $\mathrm{Na}_{3} \mathrm{Cit}$ равно $1.0: 1.8: 3.2$. Нами было изучено влияние концентраций цитрата натрия и нитрата серебра (рис. 4). Как видно на рис. $4 a$, при восстановлении $0.6 \mathrm{M}$ растворов $\mathrm{AgNO}_{3}$ с ростом концентрации стабилизатора вплоть до 1.5 М происходит закономерное снижение среднего размера частиц. Согласно рис. 46 снижение концентрации нитрата серебра до 0.4 М в присутствии 1.2 М растворов цитрата натрия приводит к постепенному снижению размера частиц, после чего выходит на плато. Таким образом, для синтеза наиболее мелких частиц минимальное необходимое отношение $\mathrm{AgNO}_{3}: \mathrm{Na}_{3}$ Сit составляет около 2.5, что на 20\% ниже, чем было предложено Кэри Ли.
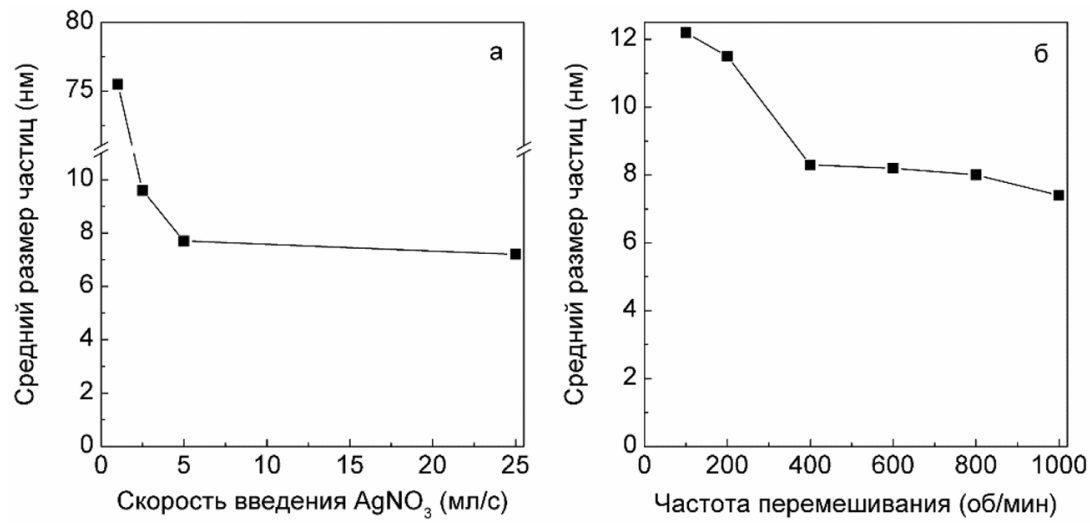

Рис. 3. Влияние скорости инжекции раствора $\mathrm{AgNO}_{3}$ (а) и (б) скорости перемешивания реакционной среды на средний размер частиц

Fig. 3. The influence of the injection rate of $\mathrm{AgNO}_{3}$ (a) and the mixing rate (б) of the reaction medium on the average particle size 

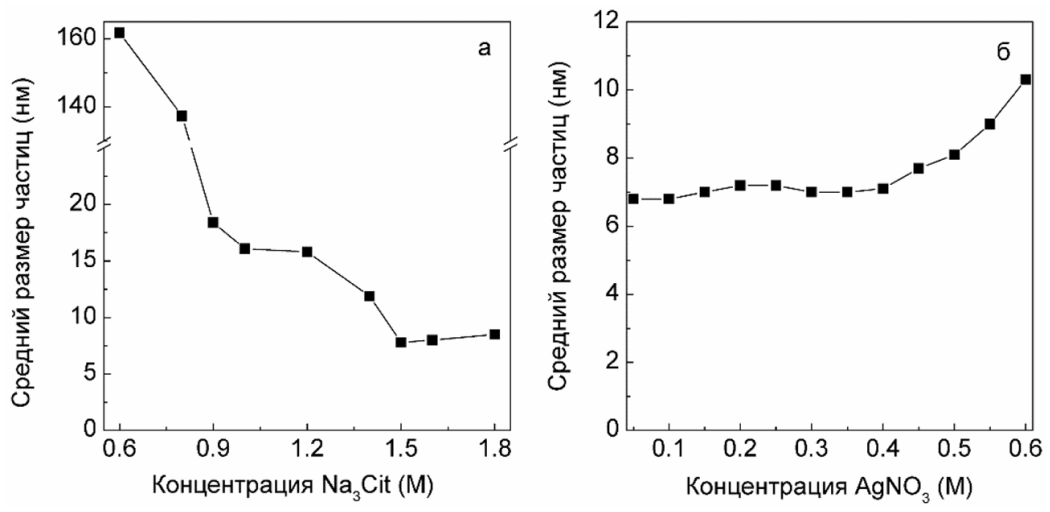

Рис. 4. Влияние концентраций цитрата натрия (а) и (б) нитрата серебра на средний размер частиц

Fig. 4. Effect of the concentration of $\mathrm{NaNO}_{3}$ (a) and $\mathrm{AgNO}_{3}(б)$ on the average particle size
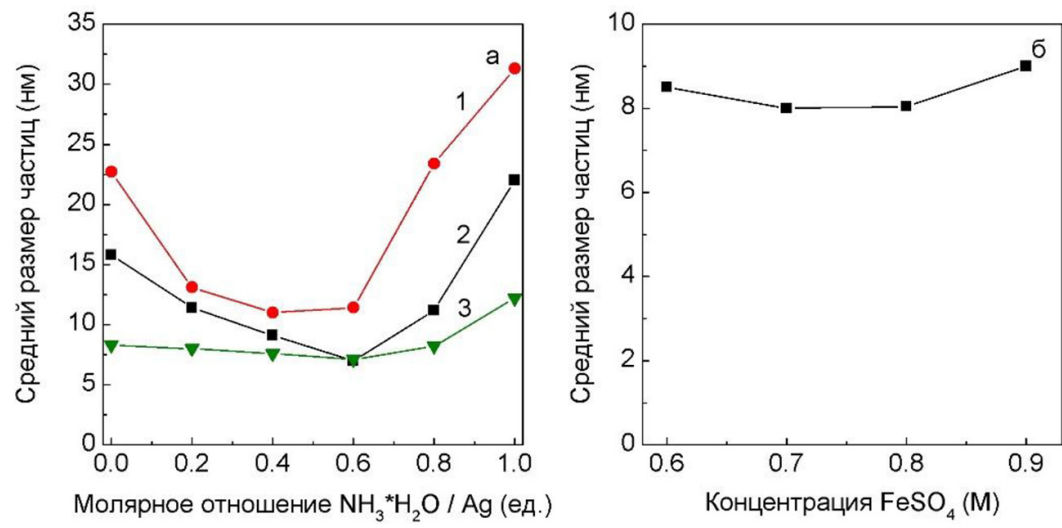

Рис. 5. Влияние молярного отношения $\mathrm{NH}_{3} \cdot \mathrm{H}_{2} \mathrm{O}: \mathrm{Ag}$ при концентрации стабилизатора $1-0.9 \mathrm{M}, 2-1.2 \mathrm{M}$ и 3 - 1.5 (а) и (б) концентрации $\mathrm{FeSO}_{4}$ на средний размер частиц

Fig. 5. Effect of molar ratio of $\mathrm{NH}_{3} \cdot \mathrm{H}_{2} \mathrm{O}:$ Ag with stabilizer concentrations of $1-0.9 \mathrm{M}, 2-1.2 \mathrm{M}, 3-1.5 \mathrm{M}$ (a) and the concentration of $\mathrm{FeSO}_{4}$ (б) on the average paricle size

Было установлено влияние добавок аммиака на размер образующихся в ходе синтеза частиц. Как видно на рис. $5 a$, средний размер частиц при повышении молярного отношения $\mathrm{NH}_{3} \cdot \mathrm{H}_{2} \mathrm{O}: \mathrm{Ag}$ снижается, проходит через минимум при значении около 0.6 , после чего снова возрастает. При этом зависимость приобретает более выраженный характер при снижении концентрации стабилизатора с 1.5 до 0.9 М. Видимо, молекулы аммиака в данной системе проявляют стабилизирующий эффект [21-24], частично замещая цитрат натрия, что наряду со снижением $\mathrm{pH}$ способствует образованию более мелких частиц [25]. Однако при повышении концентрации аммиака выше критического значения растворяются уже сформированные мелкие и наиболее химически активные частицы серебра.

На рис. $5 б$ представлена зависимость среднего размера частиц от концентрации восстановителя $\mathrm{FeSO}_{4}$. Видно, что концентрация восстановителя в диапазоне 0.6 - 0.9 М практически не влияет на средний размер частиц. Это позволяет снизить молярное отношение $\mathrm{AgNO}_{3}$ : $\mathrm{FeSO}_{4}$ почти в два раза, с 1.8 до 1.0, по сравнению с классической методикой Кэри Ли. 
В результате проведенных экспериментов были найдены оптимальные условия синтеза и выделения частиц, которые позволили снизить молярное отношение $\mathrm{AgNO}_{3}: \mathrm{FeSO}_{4}: \mathrm{Na}_{3} \mathrm{Cit}$ с $1.0: 1.8: 3.2$ до $1.0: 1.0: 2.5$ и получить при этом более тонкие и менее загрязненные частицы, чем в классической методике Кэри Ли. А именно, в оптимальном режиме в смесь растворов $\mathrm{Na}_{3} \mathrm{Cit}\left(1.5 \mathrm{M} ; 5\right.$ мл) и $\mathrm{FeSO}_{4}$ (0.6 M; 5 мл) при перемешивании на скорости 1000 об/мин вводили раствор $\mathrm{AgNO}_{3}$ (0.6 M; 5 мл) со скоростью 25.0 мл/с. Очистку полученных частиц проводили при ускорении центрифугирования $200 \mathrm{~g}$ и концентрации коагулянта $\mathrm{NaNO}_{3} 1.0 \mathrm{M}$.

По данным просвечивающей электронной микроскопии (рис. $6 a$ ) в оптимальных условиях были получены частицы со средним размером $6.5 \pm 1.8$ нм и формой, близкой к сферической. Анализ изображения микродифракции электронов SAED (рис. 6б) показал наличие рефлексов, типичных для фазы чистого металлического серебра $[4,5,9,15]$.

Анализ поверхности частиц методами РФЭС и ОЭС представлен на рис. 7. Согласно данным обзорных спектров (рис. 6a), образец содержит около $1.2 \% \mathrm{Na}$ и $0.0 \% \mathrm{Fe}$. Линия $\mathrm{Ag} 3 \mathrm{~d}$ хорошо описывается одной полосой с энергией связи при 368.3 эВ, характерной для металлического серебра [15]. Оже-спектры Ag M5N45N45 с кинетической энергией при 357.7 эВ также подтверждают, что основное состояние наночастиц $-\operatorname{Ag}^{0}[26,27]$. Таким образом, мы не обнаружили на поверхности образцов никаких следов $\mathrm{Ag}^{+}$и $\mathrm{Fe}^{3+}$ соединений, которые присутствуют, по утверждениям некоторых исследователей $[14,28,29]$. Спектр C 1s содержит компоненты, специфичные для ВОПГ (284.5 эВ), алифатического углерода (285.0 эВ), включая некоторый случайный углерод, спирта С-ОН (286.5 эВ), карбоновой группы СОО- (288.6 эВ), и слабый широкий пик при 290.7 эВ обусловлен спутником из графитового углерода [15, 30]. Стоит отметить, что энергия связи карбоксильной группы СОО- (288.6 эВ) выше, чем для таковой в соединении $\mathrm{Na}_{3} \mathrm{Cit}$ (288.6 эB), что может указывать на перенос электронной плотности на металлический остов наночастицы [26].

Также из спектра $\mathrm{C} 1 \mathrm{~s}$ видно, что отношение количества групп COO-: C-OH для полученного образца равно 1.6, вместо 3.0, как полагается для соединения $\mathrm{Na}_{3} \mathrm{Cit}$. Спектры O 1s содержат полосы $\mathrm{C}=\mathrm{O}(531.1$ эВ) в карбоксильной группе, С-О• (532.0 эВ) - в спиртовой и карбоксильной группах и для молекулярной воды (533.4 эВ и более) $[15,26,31]$. В целом спектры O 1s под-
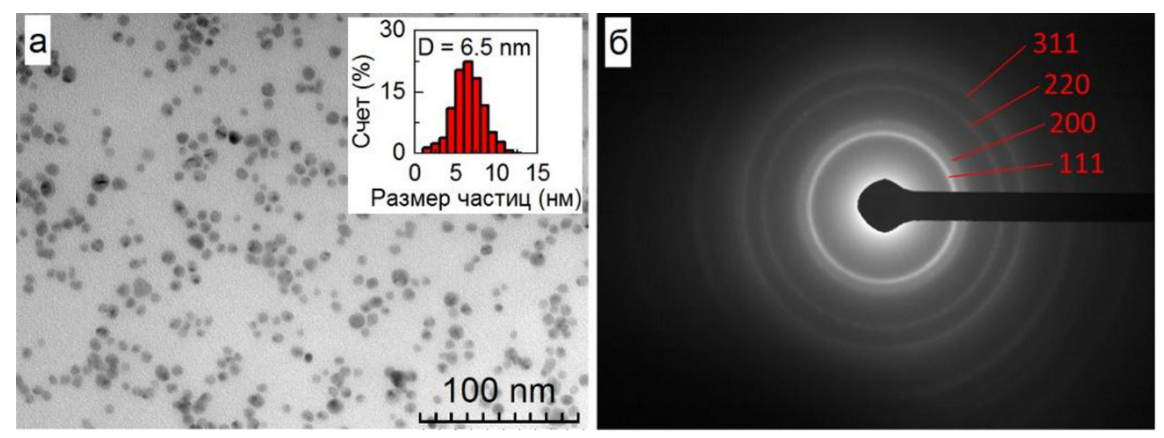

Рис. 6. Микрофотография ПЭМ с гистограммой распределения по размеру частиц (а) и электронограмма наночастиц серебра, полученных в оптимальных условиях (б)

Fig. 6. TEM images with the histogram of the size distribution of the particles (a) and electronograms of silver nanoparticles (б) 
тверждают выводы о снижении количества карбоксильных групп в $1.5-2.0$ раза по сравнению с исходным цитрат-ионом.

До сих пор было принято считать, что поверхность частиц Кэри Ли стабилизирована цитрат-ионами, однако мы показали здесь и ранее [15], что на самом деле в качестве стабилизатора выступает некоторый продукт их частичного окисления. Тем не менее вопрос о маршруте окисления цитрат-ионов и его влиянии на стабильность системы Кэри Ли остается открытым и требует дальнейшего изучения.

Спектры ИК Фурье (рис. 8) исходного цитрат натрия содержат полосы симметричного и асимметричного колебания СОО- (1592 и $\left.1399 \mathrm{~cm}^{-1}\right)$, деформационного колебания $\mathrm{COO}^{-}$(892 и $\left.838 \mathrm{~cm}^{-1}\right)$, асимметричного колебания С-ОН (1300 и $\left.1078 \mathrm{~cm}^{-1}\right)$ и растяжения С-О $(1196,1158$ и $1137 \mathrm{~cm}^{-1}$ ) [32, 33]. В спектрах полученных наночастиц полосы симметричного и асимметричного растяжения $\mathrm{COO}^{-}$сдвигаются в область 1623 и 1350 см$^{-1}$, что можно объяснить связыванием карбоксильных групп с поверхностью частиц [33-35]. Наряду с этим появление слабой широкой полосы при $1518 \mathrm{~cm}^{-1}$ может отвечать появлению по крайне мере еще одного типа связывания карбоксильных групп с поверхностью частиц [33-35]. Относительный вклад полос в диапазоне 1298 - 1031 см$^{-1}$, в основном отвечающих колебаниям С-О, становится более выраженным, что можно связать с уменьшением количества карбоксильных групп.

\section{Заключение}

С использованием специально собранной установки для синтеза в воспроизводимых условиях было изучено влияние реакционных условий на средний размер частиц серебра, образую-
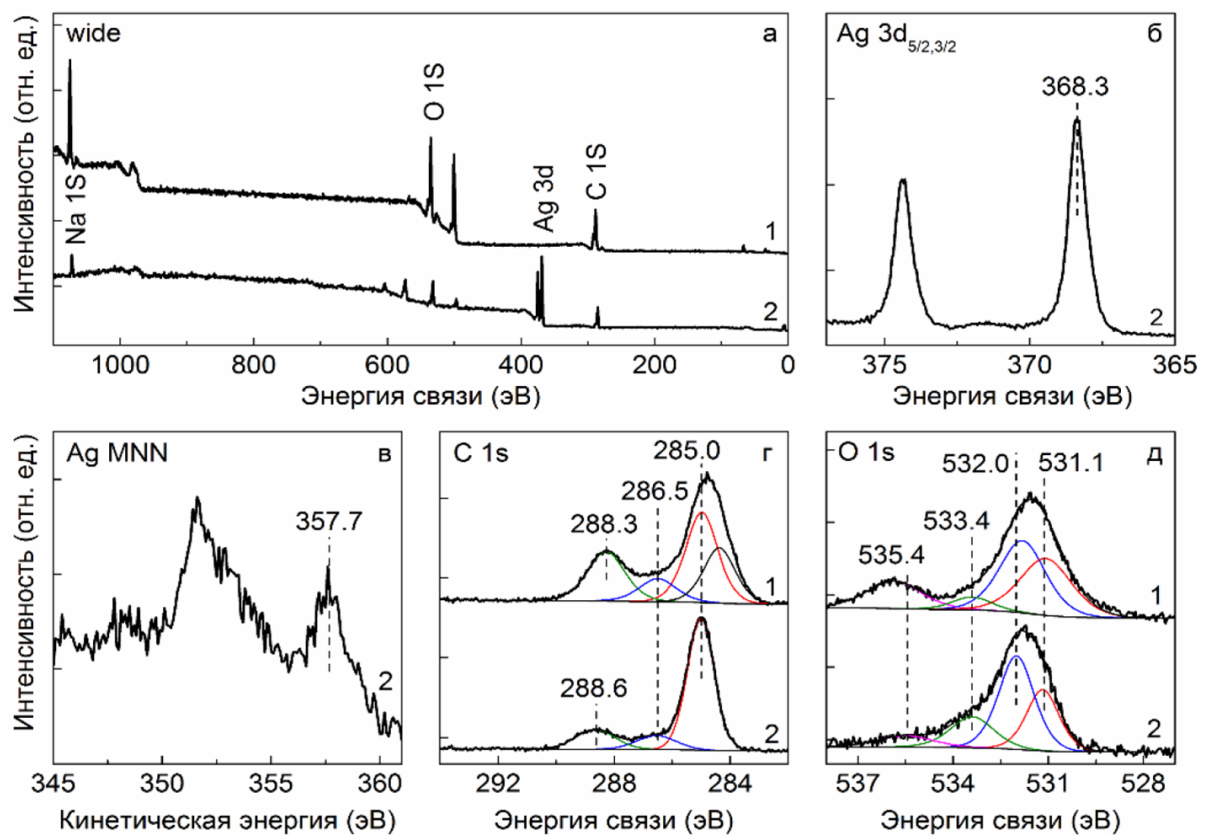

Рис. 7. Рентгеновские фотоэлектронные спектры исходного $\mathrm{Na}_{3} \mathrm{Cit} \cdot 5.5 \mathrm{H}_{2} \mathrm{O}$ (крив. 1) и (крив. 2) синтезированных в оптимальных условиях наночастиц серебра

Fig. 7. XPS spectrum of the $\mathrm{Na}_{3} \mathrm{Cit} \cdot 5.5 \mathrm{H}_{2} \mathrm{O}$ (curve 1) and (curve 2) silver nanoparticles under optimal conditions 


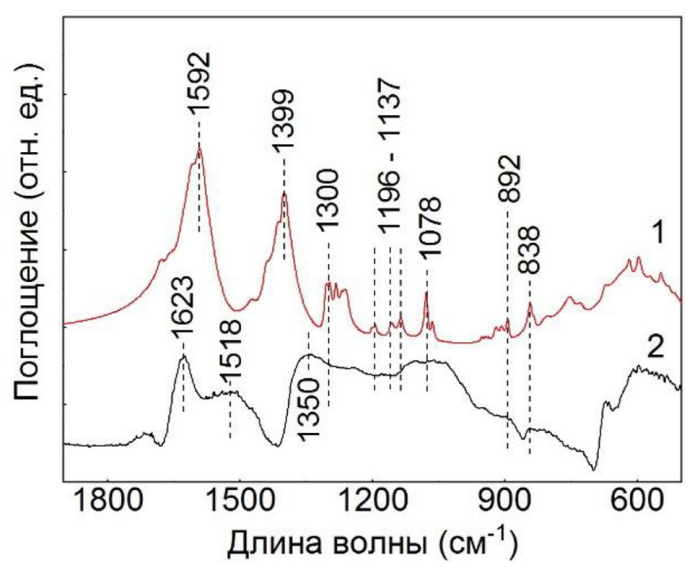

Рис. 8. ИК-Фурье-спектры $\mathrm{Na}_{3} \mathrm{Cit} \cdot 5.5 \mathrm{H}_{2} \mathrm{O}$ (крив. 1) и (крив. 2) синтезированных в оптимальных условиях наночастиц серебра

Fig. 8. FTIR spectrum of $\mathrm{Na}_{3} \mathrm{Cit} \cdot 5.5 \mathrm{H}_{2} \mathrm{O}$ (curve 1) and (curve 2) silver nanoparticles under optimal conditions

щихся при восстановлении нитрата серебра цитратным комплексом железа (II). Было показано, что для синтезированных частиц коагуляция $1.0 \mathrm{M}$ раствором $\mathrm{NaNO}_{3}$ и центрифугирование при ускорении $200 \mathrm{~g}$ приводят к минимальной агрегации при их выделении. Использование максимальных скоростей перемешивания реакционной среды (1000 об/мин) и инжекции раствоpa $\mathrm{AgNO}_{3}(25$ мл/c) позволяет получить наиболее однородные условия для синтеза наночастиц серебра. Установлено, что для получения наиболее мелких частиц минимальная концентрация стабилизатора может быть снижена до 1.5 М против $1.8 \mathrm{M}$, предложенных в классической методике Кэри Ли. Также было обнаружено, что концентрация восстановителя незначительно влияет на систему и может быть снижена до $0.6 \mathrm{M}$ против $1.08 \mathrm{M}$ в исходной рецептуре Кэри Ли. Найденные закономерности и разработанная на их основе оптимальная методика позволили снизить размер частиц, общую концентрацию реагентов на $33 \%$ и повысить чистоту продукта.

Методом ПЭМ было показано, что в оптимальных условиях были получены чистые металлические частицы со средним размером $6.5 \pm 1.8$ нм. Методом РФЭС было установлено, что поверхность частиц достаточно чиста и не содержит соединений $\mathrm{Ag}$ (I) и $\mathrm{Fe}$ (II, III).

Кроме того, было показано, что поверхность частиц стабилизирована не цитрат-ионами, как считалось ранее, а продуктом его частичного окисления. Методом ИК-Фурье-спектроскопии было подтверждено, что цитрат-ион в образце находится в частично окисленном виде и существует, по крайней мере, два вида связывания карбоксильных групп с поверхностью наночастиц.

\section{Благодарности}

Исследования были выполнены с использованием оборудования Красноярского регионального центра коллективного пользования ФИЦ КНЦ СО РАН. Работа выполнена при финансовой поддержке Российского научного фонда, грант № 18-73-00142.

We are grateful to the Krasnoyarsk Regional Center of Research Equipment of Federal Research Center «Krasnoyarsk Science Center SB RAS». This research was funded by RUSSIAN SCIENCE FOUNDATION, grant number 18-73-00142. 


\section{Список литературы / References}

1. Alarcon E.I., Griffith M., Udekwu K.I. Silver Nanoparticle Applications In the Fabrication and Design of Medical and Biosensing Devices. Springer.: New York, 2015. 157 p.

2. Welles A.E. Silver Nanoparticles: Properties, Characterization and Applications (Nanotechnologh Science and Technology). Nova Sci. Publ.: New York, 2010. 363 p.

3. Awan T., Bashir A., Tehseen A. Chemistry of Nanomaterials (Fundamentals and Applications). Elsevier.: Amsterdam, 2020. 342 p.

4. Bulavchenko A.I., Arymbaeva A.T., Demidova M.G., Popovetskiy P.S., Plyusnin P.E., Bulavchenko O.A. Synthesis and Concentration of Organosols of Silver Nanopartcles Stabilized by AOT: Emulsion vs Microemulsion. Langmuir 2018. Vol. 34(8), P. 2815-2822.

5. Popovetskiy P.S., Arymbaeva A.T., Bordzilovskiy D.S., Mayorov A.P., Maksimovskiy E.A., Bulavchenko A.I. Synthesis and Electrophoretic Concentration of Silver Nanoparticles in Water-in-Oil Emulsions of Sodium Bis(2-Ethylhexyl) Sulfosuccinate and Preparation of Conductive from Them Coatings by Selective Laser Sintering. Colloid Journal 2019. Vol. 81(4), P. 439-445.

6. Shaik A.H., Reddy D.S. Formation of 2D and 3D superlattices of silver nanoparticles inside an emulsion droplet. Materials Research Express 2017. Vol. 4(3), P. 1-9.

7. Wei C.C., Yu C.T., Chih L.Y. Encapsulated silver nanoparticles in water/oil emulsion for conductive inks. Journal of the Taiwan Institute of Chemical Engineers 2018. Vol. 92, P. 8-14.

8. Liu J., Lee J.B., Kim D.H., Kim Y. Preparation of high concentration of silver colloidal nanoparticles in layered laponite sol. Colloid Surface A 2007. Vol. 302, P. 276-279.

9. Kumar A., Aerry S., Goia D.V. Preparation of concentrated stable dispersions of uniform Ag nanoparticles using resorcinol as reductant. Journal of Colloid and Interface Science 2016. Vol. 470, P. $196-203$.

10. Lodeiro P., Achterberg E.P., Pampín J., Affatati A., El-Shahawi M.S. Silver nanoparticles coated with natural polysaccharides as models to study AgNP aggregation kinetics using UV-Visible spectrophotometry upon discharge in complex environments. Science of the Total Environment 2016. Vol. 539, P. 7-16.

11. Carey L.M. Allotropic forms of silver. Am. J. Sci.1987. Vol. 37, P. 476-491.

12. Frens G., Overbeek J.Th.G. Carey Lea's colloidal silver. Kolloid-Zeitschrift und Zeitschrift für Polymere 1969. Vol. 233 (1-2), P. 922-929.

13. Fornasiero D., Grieser F. The Kinetics of Electrolyte Induced Aggregation of Carey Lea Silver Colloids. J. Colloid Interface Sci. 1991. Vol. 141 (1), P. 168-179.

14. Jolivet J.P., Gzara M., Mazieres J., Lefebvre J. Physicochemical Study of Aggregation in Silver Colloids. J. Colloid Interface Sci. 1985. Vol. 107 (2), P. 429-441.

15. Mikhlin Y.L., Vorobyev S.A., Saikova S.V., Vishnyakova E.A., Romanchenko A.S., Zharkov S.M., Larichev Y.V. On the nature of citrate-derived surface species on Ag nanoparticles: Insights from X-ray photoelectron spectroscopy. Appl. Surf. Sci. 2018. Vol. 427, P. 687-694.

16. Li M., Xiao Y., Zhang Z., Yu J. Bimodal Sintered Silver Nanoparticle Paste with Ultrahigh Thermal Conductivity and Shear Strength for High Temperature Thermal Interface Material Applications. ACS Appl. Mater. Interfaces 2015. Vol. 7, P. 9157-9168.

17. Wang Z., Wang W., Jiang Z., Yu D. Low temperature sintering nano-silver conductive ink printed on cotton fabric as printed electronics. Prog. Org. Coat. 2016. Vol. 101, P. 604-611. 
18. Liu Z., Ji H., Wang S., Zhao W., Huang Y., Feng H., Wei J., Li M. Enhanced Electrical and Mechanical Properties of a Printed Bimodal Silver Nanoparticle Ink for Flexible Electronics. Phys. Status Solidi A 2018. Vol. 215, P. 1-9.

19. Xiao Y., Zhang Z.H., Yang M., Yang H.F., Li M.Y., Cao Y. The effect of NaOH on room temperature sintering of Ag nanoparticles for high-performance flexible electronic application. Mater. Lett. 2018. Vol. 222, P. 16-20.

20. Kim K.Y., Choi Y.T., Seo D.J., Park S.B. Preparation of silver colloid and enhancement of dispersion stability in organic solvent. Mater. Chem. Phys. 2004. Vol. 88, P. 377-382.

21. Maaz K. Silver Nanoparticles: Fabrication, Characterization and Applications. IntechOpen.: London, 2018. 275 p.

22. Buccolieri A., Serra A., Giancane G., Manno D. Colloidal solution of silver nanoparticles for label-free colorimetric sensing of ammonia in aqueous solutions. Beilstein J. Nanotechnol. 2018. Vol. 9, P. 499-507.

23. Montazer M., Shamei A., Alimohammadi F. Synthesis of nanosilver on polyamide fabric using silver/ammonia complex. Materials Science and Engineering C 2014. Vol. 38, P. 170-176.

24. Gorup L.F., Longo E., Leite E.R., Camargo E.R. Moderating effect of ammonia on particle growth and stability of quasi-monodisperse silver nanoparticles synthesized by the Turkevich method. J. Colloid Interf. Sci. 2011. Vol. 360, P. 355-358.

25. Shenashen M.A., El-Safty S.A., Elshehy E.A. Synthesis, Morphological Control, and Properties of Silver Nanoparticles in Potential Applications. Part. Part. Syst. Charact. 2014. Vol. 31, P. 293-316.

26. Mikhlin Yu.L., Vishnyakova E.A., Romanchenko A.S., Saikova S.V., Likhatski M.N., Larichev Yu.V., Tuzikov F.V., Zaikovskii V.I., Zharkov S.M. Oxidation of Ag nanoparticles in aqueous media: Effect of particle size and capping. Appl. Surf. Sci. 2014. Vol. 297, P. 75-83.

27. Kibis L.S., Stadnichenko A.I., Pajetnov E.M., Koscheev S.V., Zaykovskii V.I., Boronin A.I. The investigation of oxidized silver nanoparticles prepared by thermal evaporation and radio-frequency sputtering of metallic silver under oxygen. Appl. Surf. Sci. 2010. Vol. 257, P. 404-413.

28. Munro C.H., Smith W.E., Garner M., Clarkson J., White P.C. Characterization of the Surface of a Citrate-Reduced Colloid Optimized for Use as a Substrate for Surface-Enhanced Resonance Raman Scattering. Langmuir 1995. Vol. 11, P. 3712-3720.

29. Jolivet J.P., Gzara M., Mazieres J., Lefebvre J. Physicochemical Study of Aggregation in Silver Colloids. J. Colloid 1985. Vol. 107(2), P. 429-441.

30. Park J.W., Shumaker-Parry J.S. Structural study of citrate layers on gold nanoparticles: role of intermolecular interactions in stabilizing nanoparticles. J. Am. Chem. Soc. 2014. Vol. 136, P. 1907 1921.

31. Lopez G.P., Castner D.G., Ratner B.D. XPS O 1s Binding Energies for Polymers Containing Hydroxyl, Ether, Ketone and Ester Groups. Surface and Interface Analysis 1991. Vol. 17, P. 267-272.

32. Bichara L.C., Lan'us H.E., Ferrer E.G., Gramajo M.B., Brandan S.A. Vibrational Study and Force Field of the Citric Acid Dimer Based on the SQM Methodology. Advances in Physical Chemistry 2011. Vol. 1, P. 1-10.

33. Thottoli A.K., Unni A.K.A. Effect of trisodium citrate concentration on the particle growth of ZnS nanoparticles. Journal of Nanostructure in Chemistry 2013. Vol. 3(56), P. 1-12.

$$
-383-
$$


34. Kilin D.S., Prezhdo O.V., Xia Y. Shape-controlled synthesis of silver nanoparticles:Ab initiostudy of preferential surface coordination with citric acid. Chemical Physics Letters 2008. Vol. 458, P. 113-116.

35. Monti S., Barcaro G., Sementa L., Carravetta V., Agren H. Characterization of the adsorption dynamics of trisodium citrate on gold in water solution. RSC Adv. 2017. Vol. 7, P. 49655-49663. 